\title{
An Inhibitor of Serine Proteases, Neuroserpin, Acts as a Neuroprotective Agent in a Mouse Model of Neurodegenerative Disease
}

\author{
Yannick Simonin, ${ }^{1}$ Yves Charron, ${ }^{2}$ Peter Sonderegger, ${ }^{3}$ Jean-Dominique Vassalli, ${ }^{2}$ and Ann C. Kato ${ }^{1}$ \\ Departments of ${ }^{1}$ Basic Neurosciences and ${ }^{2}$ Genetic Medicine and Development, Faculty of Medicine, University of Geneva, CH-1211 Geneva, Switzerland, \\ and ${ }^{3}$ Institute of Biochemistry, University of Zurich, CH-8057 Zurich, Switzerland
}

\begin{abstract}
Various studies suggest that proteolytic activity may be involved in a number of neurodegenerative disorders, including stroke and seizure. In this report, we examined the role of tryptic serine proteases, plasminogen activators (PAs), in the evolution of a neurodegenerative disease. Transgenic mice overexpressing an axonally secreted inhibitor of serine proteases (neuroserpin) were crossed with mice characterized by a "dying-back" motor neuron disease [progressive motor neuronopathy ( $p m n / p m n)$ ]. Compared with pmn/pmn mice that showed an increase in PA activity, double mutant mice had decreased PA activity in sciatic nerves and spinal cord; their lifespan was increased by $50 \%$, their motor behavior was stabilized, and histological analysis revealed increased numbers of myelinated axons and rescue of motoneuron number and size. This is the first report showing that a class of serine proteases (PAs) may be involved in the pathogenesis of a motor neuron disease and more specifically in axonal degeneration. Inhibiting serine proteases could offer a new strategy for delaying these disorders.
\end{abstract}

Key words: motoneurons; axon; spinal cord; plasminogen activator; neuroserpin; pmn mice

\section{Introduction}

Proteolytic activity has been suggested to be involved in a number of neurological disorders, including stroke, seizure, and Wallerian degeneration. Recently, it has been shown that plasminogen activators (PAs) are implicated in axonal degeneration in a mouse model of multiple sclerosis, experimental allergic encephalomyelitis (Lu et al., 2002; East et al., 2005), and other studies have shown that PAs are activated during Wallerian degeneration (Bignami et al., 1982; Siconolfi and Seeds, 2001)

PAs [tissue PA (tPA) and urokinase PA (uPA)] catalyze the conversion of serum plasminogen to plasmin, the active fibrinolytic enzyme, and they are expressed in the CNS in neurons and glia. PAs have been shown to play a role in axonal elongation and nerve regeneration (Siconolfi and Seeds, 2001). It has been proposed that deregulation of the PA system could aggravate neurodegenerative processes in many pathological situations. For example, PAs and in particular tPA mediate neuronal degeneration in models of stroke, epilepsy, and in Alzheimer's disease (for review, see Melchor and Strickland, 2005).

In this report, we determined whether PAs could be involved in the neurodegenerative process of axons and cell bodies of mo-

Received July 4, 2006; accepted Sept. 7, 2006.

This work was supported by the Association Française Contre les Myopathies (France), the Institut International de Recherche en Paraplégie, and the Swiss National Science Foundation (A.C.K., J.-D.V., and P.S.) and the European Network of Excellence "NeuroNE." We thank B. King, G. Gilliéron, C. Winkler, and C. Combepine for excellent technical help and R. Madani and F. E. Perrin for reading this manuscript.

Correspondence should be addressed to Ann C. Kato, Department of Basic Neuroscience, Centre Médical Universitaire, 1 rue Michel Servet, 1211 Geneva 4, Switzerland. E-mail: ann.kato@medecine.unige.ch.

D01:10.1523/JNEUROSCI.3582-06.2006

Copyright $\odot 2006$ Society for Neuroscience ～0270-6474/06/2610614-06\$15.00/0 toneurons in a mouse model of a neurodegenerative disease called $p m n$, for progressive motoneuropathy. $p m n / p m n$ mice are characterized by a "dying-back" motor neuronopathy (Schmalbruch et al., 1991); the pathophysiology begins in the axon terminals by an unknown process, followed by a degeneration of the motoneuron cell bodies. We first show that the activity of PA is increased in sciatic nerves and spinal cord of pmn/pmn mice compared with controls. We then crossed pmn mice with transgenic mice that overexpress an inhibitor of PAs, i.e., neuroserpin. In these double mutant mice, the lifespan, the motor behavior, the number of surviving motoneurons in the spinal cord and cranial nuclei, and the number of surviving myelinated axons are increased.

\section{Materials and Methods}

Animal models

pmn mice were obtained from the laboratory of J. L. Guénet (Institut Pasteur, Paris, France). Mice that overexpressed neuroserpin $[\mathrm{TgN}$ (hsNSwt)682 line called ThyNs mice] (Cinelli et al., 2001) were crossed with $p m n /+$ to generate $p m n /+$; ThyNs progeny (F1); these mice were crossed to generate the F2 line that contained pmn/pmn;ThyNs mice. Mice genotypes were screened by PCR. All of the experimental procedures were approved by the Ethical Committee for Animal Experimentation of the Geneva Veterinary Office.

\section{Behavioral analysis}

Spontaneous locomotor activity was recorded in a photocell activity chamber (Letica model LE 8811; Bioseb, Chaville, France), and data were analyzed using the program SeDaCom32 (Bioseb).

Analysis of myelinated axons in the facial and phrenic nerves Nerves were removed, postfixed, dehydrated, sectioned, and stained with Boracs blue as described previously (Ferri et al., 2003). 


\section{Sciatic nerves}

A

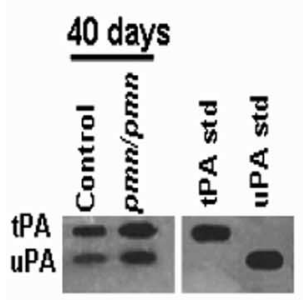

B

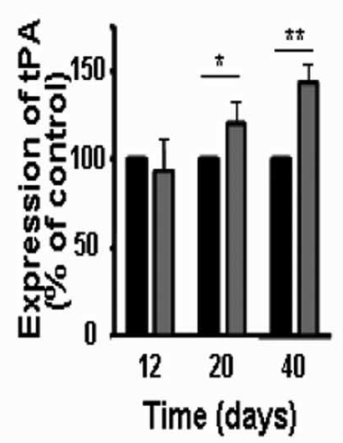

Spinal cord

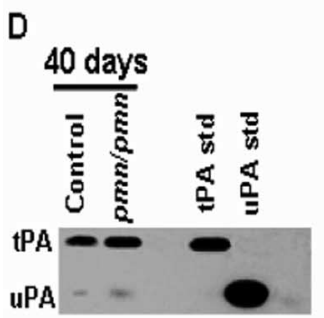

E

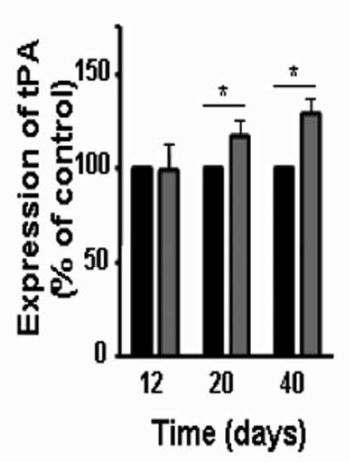

Control 口pmn/pmn

C

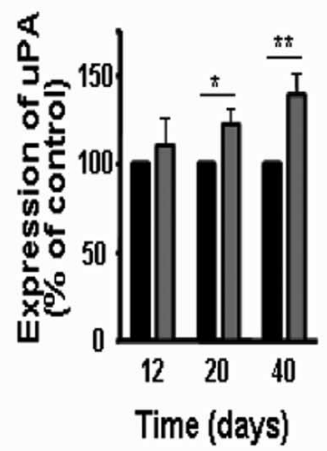

- Control םpmnipmn

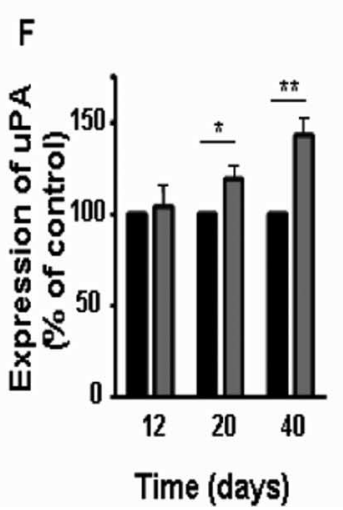

Figure 1. A, Gel zymography of PA activity in sciatic nerves of control and pmn/pmn mice at $40 \mathrm{~d}$ of age. $\boldsymbol{B}, \boldsymbol{C}$, Quantification of tPA (B) and uPA ( $C$ activities at 12,20 , and 40 d of age. $\boldsymbol{D}$, Gel zymography of PA activity in spinal cord of control and pmn/pmn mice at 40 d of age. $\boldsymbol{E}, \boldsymbol{F}$, Quantification of $\operatorname{tPA}(\boldsymbol{E})$ and uPA $(\boldsymbol{F})$ activities; values were normalized to the level of controls that were taken as $100 \%$ ( $n=3$; ANOVA analysis, $\left.{ }^{*} p<0.01,{ }^{* *} p<0.001\right)$. std, Standard. PA activity in sciatic nerves and in spinal cord was similar between $\mathrm{pmn} / \mathrm{pmn}$ mice and control mice at a presymptomatic stage ( $12 \mathrm{~d})$, whereas there was an increase in both uPA and tPA in pmn/pmn mice at a symptomatic age ( 20 and $40 \mathrm{~d}$ ).

Histological analysis of fluorescently labeled sciatic motoneurons Labeling was performed as described previously (Perrelet et al., 2004). The fluorescent retrograde tracer fluorogold (Fluorochrome, Englewood, NJ) was injected into the gastrocnemius muscle, and animals were killed 1 week later (at $40 \mathrm{~d}$ of age). The spinal cords were sectioned at 30 $\mu \mathrm{m}$ on a cryostat. Labeled motoneurons were counted in the ventral horn of the spinal cord in segments L3-L6.

\section{Counting of motoneurons}

In spinal cord. Mice were perfused at 12,20, and $40 \mathrm{~d}$ of age. The spinal cords were cryoprotected in $20 \%$ sucrose, sectioned at $14 \mu \mathrm{m}$ on a cryostat, and stained with cresyl violet.

In cranial nuclei. After perfusion the brains were removed, dehydrated in alcohol, embedded in paraffin, sectioned at $8 \mu \mathrm{m}$ on a microtome, and stained with cresyl violet.

In both cases, only motoneurons with a clearly identifiable nucleus were counted on both sides of the spinal cord in every fifth section; the values were multiplied by five to give the final estimate of the number of motoneurons.

\section{Analysis of the cell body surface of motoneurons}

The cross-sectional area of the motoneurons was measured with RoboSoftware (Palm Microlaser Technologies, Bernried, Germany).
Zymographic assay for PAs

Mice were killed and perfused with PBS/ liquemine. Sciatic nerves were removed, crushed with a Polytron, and centrifuged to eliminate remaining cell fragments. Zymographic assays were done (with $10 \mu \mathrm{g}$ of protein) according to Vassalli et al. (1984). The optical density of the bands was measured by scanning the gels and determining the number of pixels per surface area (NIH ImageJ version 1.30; Wayne Rasband, National Institutes of Health, Bethesda, MD).

\section{Western blotting}

Western blotting was performed as described previously (Perrelet et al., 2004). Primary antibodies included anti- $\beta 3$-tubulin (mouse, 1:500; Chemicon, Hofheim, Germany) and anti-actin (goat, 1:2000; Santa Cruz Biotechnology, Santa Cruz, CA). Secondary antibodies included anti-mouse and anti-goat IgG, horseradish peroxidase linked. The optical density of the bands was measured as described previously for zymography.

\section{Results}

PAs are activated in sciatic nerves and spinal cord of $p m n / p m n$ mice

The initial goal of our work was to determine whether PAs can play a role in the pathophysiology of the pmn dying-back motor neuronopathy. These mice develop muscle weakness and atrophy starting at 2 weeks of age and die at $\sim 6$ weeks of age. We first observed that PAs were differentially expressed in sciatic nerves of control and mutant mice (Fig. $1 A-C$ ). A quantitative analysis by densitometry at 12,20 , and $40 \mathrm{~d}$ of age (Fig. $1 B, C$ ) showed no difference in PA activity in $p m n / p m n$ mice and control mice at a presymptomatic stage (12 d of age), whereas it was already increased in $\mathrm{pmn} / \mathrm{pmn}$ mice at $20 \mathrm{~d}$ of age. PA activity increased even further at $40 \mathrm{~d}$ of age, corresponding to the end stage of the disease.

Differential expression of PA activity was also observed in the spinal cord; gel zymography showed that both tPA and uPA activity were higher in the spinal cord of 20- and 40-d-old pmn/pmn mice versus control mice (Fig. $1 D-F$ ). These results suggest that PAs may be candidate factors for playing a role in axonal degeneration and motoneuron loss in pmn/pmn mice. To determine which cell types overexpress PAs in the spinal cord and sciatic nerves, we performed in situ hybridization for both tPA and uPA mRNAs on sections from control and pmn/pmn mice. In spinal cords, an increase in tPA mRNA was observed in $p m n / p m n$ compared with control mice in various cell types (such as neurons and glia) and not only in motoneurons (data not shown). uPA mRNA was undetectable, probably because of the low expression of this serine protease in the spinal cord (Fig. $1 D$ ). We did not detect any labeling of UPA and tPA mRNA in sciatic nerves, suggesting that the primary site of PA synthesis was in the spinal cord (data not shown). To study whether immune cells may be involved in axonal degeneration or PA expression, we compared extracts of sciatic nerves and spinal cord from $p m n / p m n$ and control mice by 
A

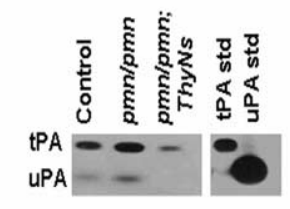

C

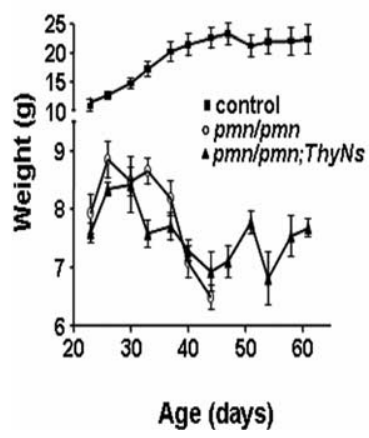

B

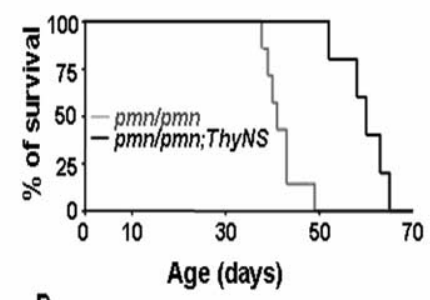

D

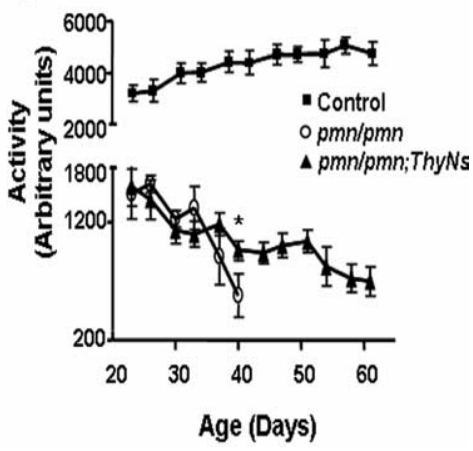

Figure 2. A, PA activity is strongly reduced in pmn/pmn;ThyNs mice. Gel zymography of PA activity in sciatic nerves of control, $p m n / p m n$, and $p m n / p m n$; ThyNs mice at $40 \mathrm{~d}$ of age. $\boldsymbol{B}-\boldsymbol{D}$, Neuroserpin overexpression extends lifespan and stabilizes motor function in $p m n / p m n$ mice. $\boldsymbol{B}$, Survival of $p m n / p m n(n=6)$ and $p m n / p m n$; ThyNs $(n=5)$ mice. The mean survival of $p m n / p m n$; ThyNs mice (bold line) is significantly increased compared with $p m n / p m n$ mice (thin line) $(p<0.001)$. C, Weight of control $(n=6), p m n / p m n(n=6)$, and pmn/pmn;ThyNs $(n=$ 6) mice. $\boldsymbol{D}$, Motor activity of control $(n=6), p m n / p m n(n=6)$, and pmn/pmn;ThyNs $(n=6)$ mice (ANOVA analysis, ${ }^{*} p<0.01$ ).

immunoblotting using an antibody (CD11) that is specific to macrophages and microglia. We found no difference in the expression of this antigen, strongly suggesting that there is no immune response to axonal degeneration in $\mathrm{pmn} / \mathrm{pmn}$ mice (data not shown).

\section{Neuroserpin overexpression partially rescued the phenotype of $p m n / p m n$ mice}

To determine whether inhibiting PAs may modify the neurodegenerative process in the $p m n$ phenotype, we crossed $p m n / p m n$ mice with ThyNs transgenic mice that overexpress an inhibitor of PAs, neuroserpin, under the control of a neuronal promoter (Thy1.2) (Cinelli et al., 2001). It has been shown previously that neuroserpin can inhibit both tPA and uPA in vivo (Yepes et al., 2000) and that it is neuronally expressed and axonally secreted (Osterwalder et al., 1998). Because PAs are also secreted proteins that function in the extracellular matrix, neuroserpin provides a tool for interfering with PA activity under pathological conditions. Because the neuroserpin and $p m n$ mutant mice were maintained in different strains (C57BL/6 and SV129, respectively), we also analyzed offspring of $(p m n /+\times \mathrm{C} 57 \mathrm{BL} / 6) \times(p m n /+\times$ $\mathrm{C} 57 \mathrm{BL} / 6)$ matings and showed that disease progression in $\mathrm{pmn} /$ pmn mice was not significantly affected by the genetic background (data not shown). No gene dosage effect was observed when comparing pmn/pmn mice carrying one or two copies of the neuroserpin transgene. By zymographic analysis, we confirmed that exogenous neuroserpin overexpression reduced PA activity in sciatic nerve extracts of $p m n / p m n$; ThyNs mice compared with $p m n / p m n$ mice (Fig. $2 A$ ).

Hindlimb atrophy and muscle weakness were evident at 2 weeks of age in $p m n / p m n$ mice, and all mice died at $\sim 40 \mathrm{~d}$ of age $(41.7 \pm 3.8 ; n=6)$. In contrast, when pmn mice were crossed

with transgenic mice overexpressing neuroserpin, the lifespan of the $p m n / p m n$ offspring carrying the ThyNs transgene was prolonged to $\sim 60 \mathrm{~d}$ of age $(61.3 \pm 5.9 ; n=5)$, i.e., an increase of $\sim 50 \%(p<0.001)$ (Fig. $2 B$ ). We did not observe a difference in the lifespan of control and ThyNs mice.

Neuroserpin overexpression did not provide a protection against the massive weight loss seen in pmn/pmn mice. The weight of the $p m n / p m n ; T h y N s$ mice stabilized to a value only slightly higher than homozygote $p m n / p m n$ before death (Fig. $2 C)$. A photocell activity chamber was used to monitor spontaneous activity (Fig. $2 D$ ). In both pmn/pmn and pmn/pmn;ThyNs mice, the activity level was dramatically lower than in controls. However, in $p m n / p m n ; T h y N s$ mice, spontaneous activity decreased more slowly and gradually compared with $\mathrm{pmn} / \mathrm{pmn}$ mice.

In previous studies, we have shown that certain factors such as ciliary neurotrophic factor act to protect both the axon and the cell body and thus increase the lifespan of pmn mice (Sagot et al., 1995a). In contrast, other molecules such as bcl-2 and glial cell line-derived neurotrophic factor were only capable of rescuing the motoneuron cell bodies without preserving the axons; under these conditions, the lifespan of the pmn mice was unaltered (Sagot et al., 1995b, 1996). In the present experiments, we hypothesized that an increase in PA levels may play a role in motoneuron degeneration, and thus inhibition of PAs by neuroserpin should protect motor axons, motoneuron cell bodies, or both. Myelinated axons were counted in the facial motor nerve of 12-, 20-, and 40-d-old pmn/pmn, pmn/pmn;ThyNs, and control littermates. At a presymptomatic age, there was no difference in the number of myelinated axons between the different groups. As expected, the number of facial axons in $p m n / p m n$ mice was strongly reduced at symptomatic ages (Fig. $3 A, B, G$ ). In contrast, axon loss was reduced in $\mathrm{pmn} / \mathrm{pmn}$; ThyNs compared with $\mathrm{pmn} /$ pmn mice (Fig. $3 B, C, G$ ).

Myelinated fibers in the phrenic nerve that innervate the diaphragm have been reported to be severely affected in $\mathrm{pmn} / \mathrm{pmn}$ mice, suggesting that these mice die by respiratory failure (Schmalbruch et al., 1991). To determine whether neuroserpin overexpression prevented this loss, myelinated axons were counted in the phrenic motor nerve at the end stage of disease (40 $\mathrm{d}$ of age) in $p m n / p m n, p m n / p m n ; T h y N s$, and control littermates. As described previously (Sagot et al., 1995b; Ferri et al., 2003), the number of phrenic axons was decreased by $45 \%$ in $\mathrm{pmn} / \mathrm{pmn}$ mice; this loss was partially prevented in neuroserpin overexpressing $p m n / p m n$ mice (Fig. 3I).

The $p m n$ disease results from a mutation in the tubulin chaperone $\mathrm{E}$ ( $t b c e$ ) gene that leads to a decreased number of microtubules in the axons (Martin et al., 2002). To determine whether neuroserpin overexpression could stabilize microtubules, we examined the level of $\beta 3$-tubulin as a quantitative index of microtubule abundance. In the sciatic nerve, at $40 \mathrm{~d}$ of age and as determined by Western blotting, the level of $\beta 3$-tubulin was decreased by $76 \%$ in $p m n / p m n$ mice compared with controls (Fig. $3 \mathrm{H}$ ), whereas in the presence of neuroserpin overexpression, it was decreased by only 55\%. Thus, neuroserpin may contribute to stabilizing or restoring the polymerization of microtubules.

Because $p m n / p m n$ mice are characterized by a loss of motoneurons, we examined whether overexpression of neuroserpin could prevent neuronal degeneration. Motoneurons in the lumbar spinal cord and in four cranial nuclei (oculo/trochlear, trigeminal, facial, and hypoglossal) were counted. Gastrocnemius motoneurons in the spinal cord were selectively labeled by retrograde transport after injection of fluorogold into the gastrocne- 

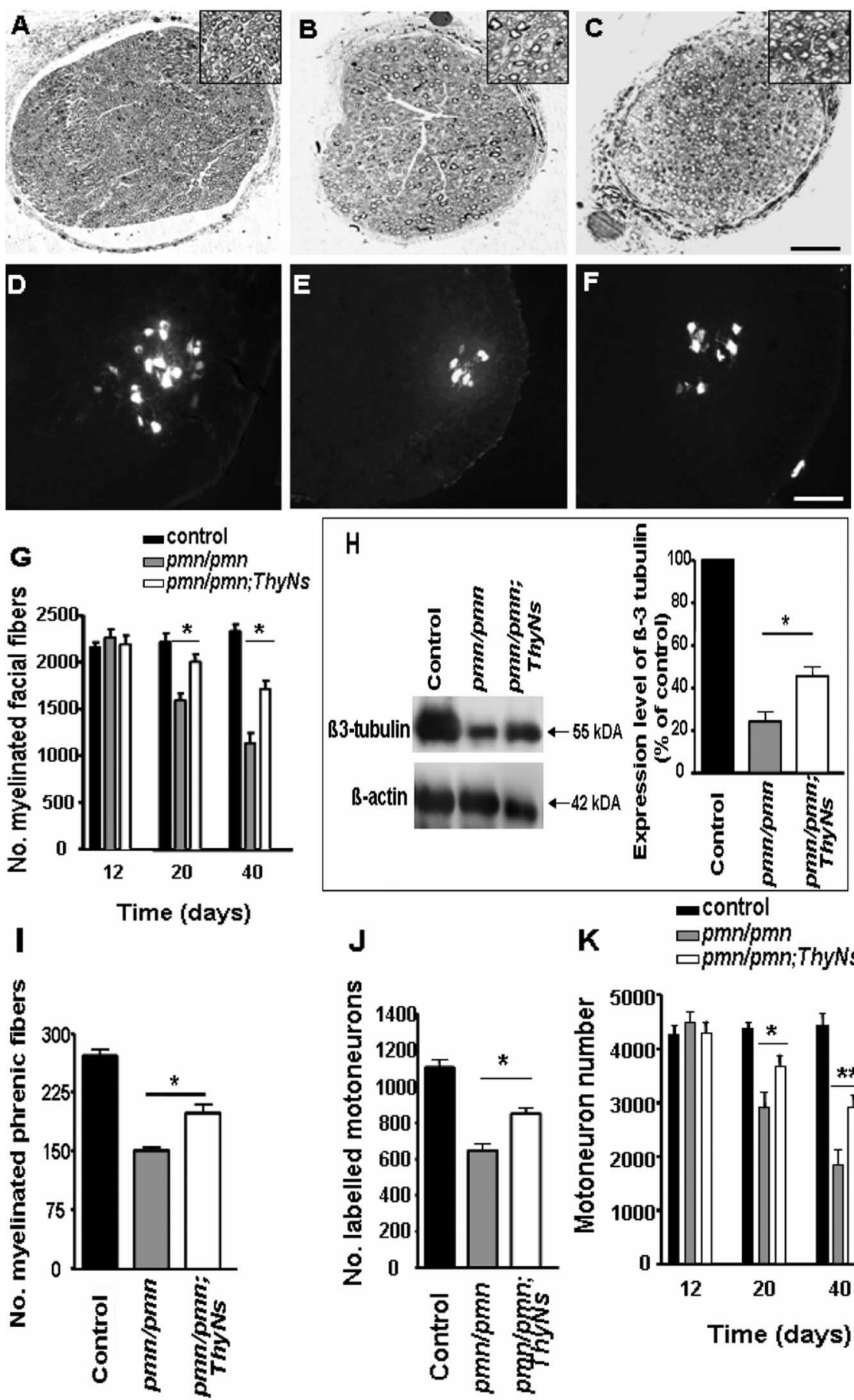

$\mathbf{K}$

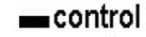

$\square p m n / p m n$ ๑pmn/pmn;ThyNs

Figure 3. Neuroserpin overexpression partially protects motor axon and motoneuron loss in pmn/pmn mice. $\boldsymbol{A}-\boldsymbol{C}$, Cross sections of facial nerves in control $(\boldsymbol{A}), \mathrm{pmn} / \mathrm{pmn}(\boldsymbol{B})$, and pmn/pmn;ThyNs ( $\boldsymbol{C}$ mice at $40 \mathrm{~d}$ of age. Insets, Threefold magnification. In $\boldsymbol{B}$, there are fewer myelinated facial nerves, and they are less compact than in $\boldsymbol{A}$ or $\boldsymbol{C}$. $\boldsymbol{D}-\boldsymbol{F}$, Transverse sections of lumbar spinal cords from control $(\boldsymbol{D}), p m n / p m n(\boldsymbol{E})$, and pmn/pmn;ThyNs $(\boldsymbol{F})$ mice after fluorogold labeling of motoneurons. $\boldsymbol{G}$, Counts of myelinated axons in facial nerves at 12,20, and $40 \mathrm{~d}$ of age. In pmn/pmn; ThyNs mice $(n=5)$, the number of myelinated axons was significantly increased (ANOVA analysis, ${ }^{*} p<0.01$ ) compared with $p m n / p m n(n=4)$. $\boldsymbol{H}$, Western blot for $\beta 3$-tubulin in sciatic nerves of control, $p m n / p m n$, and $p m n / p m n$;ThyNs mice at $40 \mathrm{~d}$ of age and quantification $(n=3)$. Values were normalized to $\beta$-actin; controls were taken as $100 \%$ (ANOVA analysis, ${ }^{*} p<0.01$ ). I, Counts of myelinated axons in phrenic nerves at $40 \mathrm{~d}$ of age. In $p m n / p m n$; ThyNs mice $(n=3)$, the number of myelinated axons was significantly increased (ANOVA analysis, ${ }^{*} p<0.01$ ) compared with $p m n / p m n$ mice $(n=3)$.J, Counts of fluorogold-labeled motoneurons at 40 d of age. In pmn/pmn; ThyNs mice $(n=$ 4), the number of fluorogold-labeled motoneurons was significantly increased (ANOVA analysis, ${ }^{*} p<0.01$ ) compared with pmn/pmn mice $(n=4) . K$, Counts of cresyl violet-labeled motoneurons in the lumbar spinal cord at 12,20 , and $40 \mathrm{~d}$ of age ( $n=$ 3) (ANOVA analysis, ${ }^{*} p<0.01,{ }^{* *} p<0.001$ ). Scale bars: $\boldsymbol{A}-\boldsymbol{C}, 50 \mu \mathrm{m} ; \boldsymbol{D}-\boldsymbol{F}, 100 \mu \mathrm{m}$. mius muscle (Fig. $3 D-F, J$ ), and the total number of lumbar spinal cord motoneurons were determined after cresyl violet staining (Fig. $3 K$ ). Whereas spinal motoneurons degenerated in $p m n / p m n$ mice, neuroserpin overexpression partially prevented the decrease in neuron number $(\sim 50 \%$ reduction in neuronal loss compared with $p m n / p m n$ mice at $40 \mathrm{~d}$ of age) (Fig. $3 E, F, J, K$ ). Similarly, whereas four populations of motoneurons in cranial nuclei were decreased in $p m n / p m n$ mice, neuroserpin overexpression partially prevented the decrease in the number of both oculo/trochlear and facial nuclei motoneurons (Fig. $4 A$ ) and in the size of facial motoneurons (Fig. $4 B-E$ ).

\section{Discussion}

In this study, we examined the role of PAs, a class of serine proteases, in axonal degeneration and motoneuron loss in the pmn mouse. The mutation in pmn mouse has been localized recently to a tubulin chaperone E gene (tbce) (Bommel et al., 2002; Martin et al., 2002). A mutation of human tbce is responsible for hypoparathyroidismretardation-dysmorphism syndrome that comprises neurological symptoms (Parvari et al., 2002). Interestingly, a recent study shows that human giant axonal neuropathy, an autosomal recessive neurodegenerative disorder, is related to the degradation of tubulin folding cofactor B (Wang et al., 2005). Thus, pmn mice may provide a useful model for studying diseases related to tubulin modification and not only to motoneuron disease.

The goal of our work is to determine whether PAs can play a role in the pathology of the pmn mouse model. We first observe an increase in PA activity at a symptomatic stage (20 and $40 \mathrm{~d}$ of age) in sciatic nerves and spinal cord of $p m n / p m n$ mice compared with control mice. No difference in PA activity is observed at a presymptomatic stage, suggesting that PA activation may be acting primarily at a later stage of the disease.

To understand whether this activation of PAs is relevant to mechanisms implicated in axonal degeneration and motoneuron loss, we determined whether the overexpression of an inhibitor of PAs, neuroserpin, could affect the disease progression and symptoms in $p m n / p m n$ mice. Therefore, we crossed pmn/pmn mice with neuroserpin overexpressing mice (ThyNS under the control of a neuronal promoter Thy 1.2) and observed an inhibition of PA activity in sciatic nerves of $p m n / p m n$; ThyNs mice by zymographic analysis, demonstrating that neuroserpin overex- 
pression inhibits PA activity in axons. The apparition of symptoms in both $p m n / p m n$ mice and in $p m n / p m n ; T h y N s$ mice occurs at 2 weeks of age, but we observed a $50 \%$ increase in the lifespan of $p m n / p m n ; T h y N s$ compared with $p m n / p m n$ mice. Furthermore, the body weight and the motor activity are stabilized at the end stage of disease. These results suggest that PAs play a deleterious role at later stages of the disease and that inhibition by neuroserpin can delay the disease progression and increase the lifespan of $p m n / p m n$ mice.

Moreover, we show that neuroserpin overexpression partially protects myelinated motor axons of facial and phrenic nerves and protects cranial motoneurons in the oculo/trochlear and facial nuclei as well as motoneurons in the spinal cord of pmn/pmn mice. Cranial motoneurons also exhibit a selective vulnerability in different mouse models of amyotrophic lateral sclerosis and in human patients; a selective protection of cranial nuclei has been observed previously in $p m n / p m n$; Wlds mice (Ferri et al., 2003). A number of hypotheses have been put forward to explain why motoneurons do not display a uniform degeneration, including a differential expression of glutamate receptors and glutamate transporter subtypes. Motoneurons in different cranial nuclei may also exhibit a differential sensitivity to PAs and/or neuroserpin or a differential level of expression of these genes.

Several mechanisms have been proposed to explain the role of PAs in axonal degeneration. PAs may promote demyelination because plasmin can directly degrade myelin basic proteins (Cammer et al., 1978). Furthermore, plasmin is the key initiator of the matrix metalloproteinase (MMP) activation cascade and has been documented to play an important role in the breakdown of myelin membranes (for review, see Cuzner and Opdenakker, 1999). Moreover MMP7, MMP9, and MMP12 are activated in optic nerve axons during Wallerian degeneration (Hughes et al., 2002). PAs could also play a direct role in apoptosis of motoneurons. Indeed, it has been shown that tPA plays a role in neuronal apoptosis (Flavin et al., 2000) and that neuroserpin protects neurons from ischemia-induced death (for review, see Galliciotti and Sonderegger, 2006). One prevailing hypothesis is that proteolytic disruption of the basement membrane by tPA may be the trigger that initiates apoptosis. Thus, the capacity of neuroserpin to block tPA-induced degradation of the basement membrane may explain the ability of neuroserpin to reduce neuronal death.

Interestingly, in our mouse model, we observed that neuroserpin overexpression partially rescues the microtubule loss in sciatic nerves of $\mathrm{pmn} / \mathrm{pmn}$ mice. It has been shown previously that tPA can play a role in microtubule destabilization in hippocampal neurons (Medina et al., 2005). Together, these results suggest that tPA could play a role in the microtubule loss observed in $p m n / p m n$ mice and that neuroserpin overexpression may protect $p m n / p m n$ mice by controlling tPA and its effect on microtubule abundance. Several studies have shown that tPA can play a protective role in axonal regeneration and functional recovery after sciatic nerve injury (Siconolfi and Seeds, 2001; Zou et al., 2006). These results are not incompatible with our own ob- servations because, in experimental paradigms using nerve crush, it is evident that extracellular debris must be removed by some type of proteolytic system before axonal regeneration can occur. Our results favor the implication of PAs in axonal degeneration in a dying-back motor neuronopathy and possibly in the pathogenesis of motoneuron degeneration. Similarly, it has been shown that PAs are implicated in axonal degeneration in a mouse model of multiple sclerosis, experimental allergic encephalomyelitis (Lu et al., 2002; East et al., 2005). Thus, PAs could be implicated in axonal degeneration under different pathological conditions.

It has been shown previously that UPA is activated in wobbler mouse muscle, another mouse model of motoneuron disease (Blondet et al., 1992), but our results are the first to implicate PAs in axonal degeneration and motoneuron loss in a dying-back motor neuronopathy. More importantly, they also show that increased expression of an inhibitor of these enzymes affects the outcome of the disease. It will be interesting to determine whether the attenuation of PA activity may offer a new strategy for delaying axonal degeneration and motoneuron loss in neurodegenerative disorders.

\section{References}

Bignami A, Cella G, Chi NH (1982) Plasminogen activators in rat neural tissues during development and in Wallerian degeneration. Acta Neuropathol 58:224-228.

Blondet B, Barlovatz-Meimon G, Festoff BW, Soria C, Soria J, Rieger F, Hantai D (1992) Plasminogen activators in the neuromuscular system of the wobbler mutant mouse. Brain Res 580:303-310.

Bommel H, Xie G, Rossoll W, Wiese S, Jablonka S, Boehm T, Sentdner M (2002) Missense mutation in the tubulin-specific chaperone E (Tbce) gene in the mouse mutant progressive motor neuronopathy, a model of human motoneuron disease. J Cell Biol 159:563-569.

Cammer W, Bloom BR, Norton WT, Gordon S (1978) Degradation of basic protein in myelin by neutral proteases secreted by stimulated macro- 
phages: a possible mechanism of inflammatory demyelination. Proc Natl Acad Sci USA 75:1554-1558.

Cinelli P, Madani R, Tsuzuki N, Vallet P, Arras M, Zhao CN, Osterwalder T, Rulicke T, Sonderegger P (2001) Neuroserpin, a neuroprotective factor in focal ischemic stroke. Mol Cell Neurosci 18:443-457.

Cuzner ML, Opdenakker G (1999) Plasminogen activators and matrix metalloproteases, mediators of extracellular proteolysis in inflammatory demyelination of the central nervous system. Neuroimmunology 94:1-14.

East E, Baker D, Pryce G, Lijnen HR, Cuzner ML, Gveric D (2005) A role for the plasminogen activator system in inflammation and neurodegeneration in the central nervous system during experimental allergic encephalomyelitis. Am J Pathol 167:545-554.

Ferri A, Sanes JR, Coleman MP, Cunningham JM, Kato AC (2003) Inhibiting axon degeneration and synapse loss attenuates apoptosis and disease progression in a mouse model of motoneuron disease. Curr Biol 13:669-673.

Flavin MP, Zhao G, Ho LT (2000) Microglial tissue plasminogen activator (tPA) triggers neuronal apoptosis in vitro. Glia 29:347-354.

Galliciotti G, Sonderegger P (2006) Neuroserpin. Front Biosci 11:33-45.

Hughes PM, Wells GM, Perry VH, Brown MC, Miller KM (2002) Comparison of matrix metalloproteinase expression during Wallerian degeneration in the central and peripheral nervous systems. Neuroscience 113:273-287.

Lu W, Bhasin M, Tsirka SE (2002) Involvement of tissue plasminogen activator in onset and effector phases of experimental allergic encephalomyelitis. J Neurosci 22:10781-10789.

Martin N, Jaubert J, Gounon P, Salido E, Haase G, Szatanik M, Guenet JL (2002) A missense mutation in Tbce causes progressive motor neuronopathy in mice. Nat Genet 32:443-447.

Medina MG, Ledesma MD, Dominguez JE, Medina M, Zafra D, Alameda F, Dotti CG, Navarro P (2005) Tissue plasminogen activator mediates amyloid-induced neurotoxicity via Erk1/2 activation. EMBO J 24:1706-1716.

Melchor JP, Strickland S (2005) Tissue plasminogen activator in central nervous system physiology and pathology. Thromb Haemost 93:655-660.

Osterwalder T, Cinelli P, Baici A, Pennella A, Krueger SR, Schrimpf SP, Meins M, Sonderegger P (1998) The axonally secreted serine proteinase inhibitor, neuroserpin, inhibits plasminogen activators and plasmin but not thrombin. J Biol Chem 273:2312-2321.

Parvari R, Hershkovitz E, Grossman N, Gorodischer R, Loeys B, Zecic A,
Mortier G, Gregory S, Sharony R, Kambouris M, Sakati N, Meyer BF, Al Aqeel AI, Al Humaidan AK, Al Zanhrani F, Al Swaid A, Al Othman J, Diaz GA, Weiner R, Khan KT, et al. (2002) Mutation of TBCE causes hypoparathyroidism-retardation-dysmorphism syndrome and autosomal recessive Kenny-Caffey Syndrome. Nat Genet 32:448-452.

Perrelet D, Perrin FE, Liston P, Korneluk RG, MacKenzie A, Ferrer-Alcon M, Kato AC (2004) Motoneuron resistance to apoptotic cell death in vivo correlates with the ratio between X-linked inhibitor of apoptosis proteins (XIAPs) and its inhibitor, XIAP-associated factor 1. J Neurosci 24:3777-3785.

Sagot Y, Tan SA, Baetge E, Schmalbruch H, Kato AC, Aebischer P (1995a) Polymer encapsulated cell lines genetically engineered to release ciliary neurotrophic factor can slow down progressive motor neuronopathy in the mouse. Eur J Neurosci 7:1313-1322.

Sagot Y, Dubois-Dauphin M, Tan SA, de Bilbao F, Aebischer P, Martinou JC, Kato AC (1995b) Bcl-2 overexpression prevents motoneuron cell body loss but not axonal degeneration in a mouse model of a neurodegenerative disease. J Neurosci 15:7727-7733.

Sagot Y, Tan SA, Hammang JP, Aebischer P, Kato AC (1996) GDNF slows loss of motoneurons but not axonal degeneration or premature death of pmn/pmn mice. J Neurosci 16:2335-2341.

Schmalbruch H, Jensen HJ, Bjaerg M, Kamieniecka Z, Kurland L (1991) A new mouse mutant with progressive motor neuronopathy. J Neuropathol Exp Neurol 50:192-204.

Siconolfi LB, Seeds NW (2001) Mice lacking tPA, uPA, or plasminogen genes showed delayed functional recovery after sciatic nerve crush. J Neurosci 21:4348-4355.

Vassalli JD, Dayer JM, Wohlwend A, Belin D (1984) Concomitant secretion of prourokinase and of a plasminogen activator-specific inhibitor by cultured human monocytes-macrophages. J Exp Med 159:1653-1668.

Wang W, Ding J, Allen E, Zhu P, Zhang L, Vogel H, Yang Y (2005) Gigaxonin interacts with tubulin folding cofactor $\mathrm{B}$ and controls its degradation through the ubiquitin-proteasome pathway. Curr Biol 15:2050-2055.

Yepes M, Sandkvist M, Wong MK, Coleman TA, Smith E, Cohan SL, Lawrence DA (2000) Neuroserpin reduces cerebral infarct volume and protects neurons from ischemia-induced apoptosis. Blood 96:569-576.

Zou T, Ling C, Xiao Y, Tao X, Ma D, Chen ZL, Strickland S, Song H (2006) Exogenous tissue plasminogen activator enhances peripheral nerve regeneration and functional recovery after injury in mice. J Neuropathol Exp Neurol 65:78-86. 